\title{
Perception Paysanne Des Effets Du Changement Climatique Sur La Production Des Noix D'anacardier (Anacardium Occidentale L.) Dans La Commune De Savalou Au Bénin
}

\author{
Tchétangni Y. A \\ Assogbadjo A. E \\ Houéhanou T
}

Laboratoire d'écologie appliquée, Faculté des Sciences agronomiques, Université d'Abomey-Calavi, BENIN, Cotonou

\section{Bello D. $O$}

Unité de Recherche Gestion Intégrée des Sols et des Cultures, Laboratoire des Sciences du Sol, Ecole des Sciences et Techniques de

Production Végétale, Faculté des Sciences Agronomiques,

Université d’Abomey-Calavi, BENIN, Cotonou

doi: 10.19044/esj.2016.v12n14p220 URL:http://dx.doi.org/10.19044/esj.2016.v12n14p220

\section{Abstract}

This study aims to analyze the perception of farmers from the effects of climate change on the production of cashew nuts in the town of Savalou. Thus 210 farmers were surveyed in the Municipality of Savalou based on a semi-structured questionnaire. The data collected relate to the socio-cultural characteristics of the planters, their perception of the causes and weather events affecting the performance of the cashew tree and adaptation strategies. The results show that the majority of farmers have age less than 50 years. The age of the plantations, the average yield, density and performance achieved in 2014 varied significantly $(\mathrm{P}<0.05)$ from one district to another. Adults and youth sociocultural groups Nago, Mahi, and Ife have seen that climate change is the major constraint in the production of cashew nuts. As for the main climatic parameter affecting the production of cashew, the majority of farmers (80\%) in this case, old Ife, Mahi and youth Idaatcha think it is rather the rainfall deficit. Regarding the causes of decline in yield, the analysis shows that the old Mahi and Ife have the perception that the prolonged drought, high winds and wildfires are the main while Idaatcha adults perceived that harmattan and wandering animals are the causes of yield loss. Ploughing followed by mowing in the late rainy season is the main coping strategy to mitigate the effects of climate change on the 
plantations of cashew. The contribution of research would be to put at the disposal of farmers planting material adapted to climate change.

Keywords: Cashew tree, climate change, farmers' perceptions, adaptation practices, Savalou

\section{Résumé}

La présente étude vise à analyser la perception des planteurs des effets du changement climatique sur la production des noix d'anacardier dans la commune de Savalou. Ainsi 210 planteurs ont été enquêtés dans la Commune de Savalou à base d'un questionnaire semi structuré. Les données collectées ont trait aux caractéristiques socio-culturelles des planteurs, à leur perception des causes et évènements climatiques affectant le rendement de l'anacardier et aux stratégies d'adaptation. Les résultats montrent que la majorité des planteurs ont un âge inférieur à 50 ans. L'âge des plantations, le rendement moyen, la densité et le rendement obtenu en 2014 varient significativement $(\mathrm{P}<0,05)$ d'un arrondissement à un autre. Les adultes et les jeunes des groupes socioculturels Nago, Mahi, et Ifè ont perçu que les changements climatiques constituent la contrainte majeure de la production des noix d'anacardier. Quant au principal paramètre climatique affectant la production d'anacardier, la majorité des planteurs (80\%) en l'occurrence les vieux Ifè et Mahi et les jeunes, Idaatcha pensent que c'est plutôt le déficit pluviométrique. En ce qui concerne les causes de baisse de rendement, l'analyse montre que les vieux Mahi et Ifè ont la perception que la sécheresse prolongée, les vents violents et les feux de végétation en sont les principales alors que les adultes Idaatcha ont perçu que l'harmattan et la divagation des animaux constituent les causes de baisse de rendement. Le labour suivi du fauchage à la fin des saisons pluvieuses est la principale stratégie d'adaptation pour atténuer les effets des changements climatiques dans les plantations d'anacardier. La contribution de la recherche serait de mettre à la disposition des planteurs du matériel végétal adapté aux changements climatiques.

Mots clés : Anacardium occidentale, changements climatiques, perception paysanne, méthodes d'adaptations, Savalou

\section{Introduction}

L'Afrique est un continent faiblement émetteur de Gaz à Effets de Serre (GES) et pourtant elle sera sans doute la plus touchée par les effets du changement climatique (Tinlot, et al., 2010). Ces changements climatiques ont entrainé naturellement un changement dans la variabilité des paramètres climatiques comme la température, l'humidité relative, la tension des 
vapeurs (Mamoudou, 2012) et le changement de régime pluviométrique. Le changement de régime pluviométrique accroît le risque de déficit hydrique pour les cultures pluviales (Métangbo, 2007).

La perception paysanne est une approche qui prend en compte les savoirs locaux des communautés à la base du phénomène des changements climatiques (GDAA, 2011). Les producteurs perçoivent les changements climatiques comme de fréquentes sécheresses, des changements des saisons, des vagues de chaleur. Les impacts du changement climatique sur l'agriculture sont perçus au niveau des perturbations saisonnières, avec comme conséquences: la perte de la production, les inondations des cultures, la sécheresse et les tensions sociales; d'où la nécessité de prendre en compte ces perturbations saisonnières (GDAA, 2011). Ces perturbations saisonnières ont des influences sur la production agricole en général et sur les plantations en particulier et seraient à la base de baisse de rendement des noix d'anacardier au Bénin. Il est donc important pour les acteurs de l'agriculture de bien connaître les effets des évolutions climatiques déjà observées et futures (RAC-F, 2014).

La filière anacarde est pour le Bénin une alternative économiquement intéressante qui représente le deuxième produit agricole d'exportation du pays après le coton (Aïvodji et al., 2009) mais se trouve être confronté aux variabilités climatiques. Le Bénin se hisse ainsi parmi les dix premiers producteurs mondiaux avec $2 \%$ de la production. Le pays a occupé en 2011 la sixième place au plan mondial avec environ 3,8 \% du volume de noix produites et le troisième rang des pays ouest-africains après le Nigéria et la Cote d'Ivoire (FAO, 2014). La culture de l'anacarde offre plusieurs opportunités tant nationales qu'internationales (Adegbola et al., 2005). Mais la variabilité climatique engendre la réduction de ces nombreux avantages à travers la baisse de la production. Selon Yabi, (2008), les problèmes de chute de fleurs et de fruits restent une préoccupation majeure des planteurs d'anacardiers et seraient dus à deux variables (l'indice de stress agroclimatique et la pluviométrie bimestrielle). Ce phénomène de variations du temps et des saisons est devenu davantage l'objet de préoccupations de tous (Jayathilaka, et al., 2012). Plusieurs facteurs pourraient expliquer ce phénomène à savoir, les conditions climatiques défavorables (sévérité de l'harmattan, déficit hydrique, vents violents). Ces conditions climatiques défavorables constituent les difficultés généralement formulées par les planteurs d'anacardiers à diverses occasions d'échanges sur la filière et nécessitent que des investigations plus approfondies soient faites pour un meilleur contrôle du phénomène (Tandjiekpon, et al., 2008). Les producteurs en contact direct avec les éléments de la nature dans leur environnement physique et biologique (Tidjani et Akponikpe, 2012) jouent un rôle actif dans le développement des technologies. Gnanglè (2012) a examiné comment les 
producteurs perçoivent le changement climatique. Plusieurs études ont montré que les perceptions des producteurs de la variabilité climatique correspondent aux tendances scientifiques des données climatiques (Gnanglè, 2012). Selon les producteurs d’anacardiers du Centre Bénin, les facteurs climatiques affectent la productivité de l'anacardier (Djènontin, 2013). Mais une meilleure compréhension de la façon dont les producteurs perçoivent la variabilité des facteurs climatiques et leurs effets sur la production de l'arbre est nécessaire afin de rechercher des méthodes d'adaptations durables. L'objectif général de cette étude est d'analyser la perception des planteurs des effets du changement climatique sur la production des noix d'anacardier dans la commune de Savalou. De façon spécifique, elle vise à 1) analyser les perceptions des planteurs sur les causes et évènements climatiques affectant la production d'anacardier dans la commune de Savalou; 2) analyser l'efficience des méthodes paysannes d'atténuation des effets du changement climatique sur le rendement d'anacardier dans la commune de Savalou; 3) déterminer les variations socioculturelles des dites perceptions des planteurs d'anacardier sur les effets du changement climatique et sur les méthodes paysannes d'adaptation dans la commune de Savalou au Bénin.

\section{Materiels Et Methodes Milieu d'étude}

La commune de Savalou est située dans le département des Collines qui appartient intégralement à la zone de climat soudano guinéen marqué par deux saisons de pluies qui couvrent les périodes d'avril à juillet et d'octobre à novembre (Balogoun et al., 2014) et deux saisons sèches (de Décembre à Mars et le mois d’Août) (PDC, 2012). Elle est comprise entre $7^{\circ} 35$ et $8^{\circ} 13$ de latitude Nord d'une part et $1^{\circ} 30$ et $2^{\circ} 6$ de longitude Est d'autre part. Elle partage ses frontières avec les communes de Dassa-Zoumè et de Glazoué à l'Est; de Djidja au Sud, de Bantè au Nord et la République du Togo à l'Ouest sur environ $65 \mathrm{~km}$ (limite Nord-sud) (Figure1). Elle s'étend sur près de 58 $\mathrm{km}$ de l'Ouest à l'Est et couvre une superficie de $2.674 \mathrm{~km}^{2}$; soit 2,37\% du territoire national (PDC, 2005).

Selon Yabi et al. (2013), la température moyenne annuelle dans ce secteur est de $27,35^{\circ} \mathrm{C}$. La moyenne annuelle de la pluviométrie est de 1124 , $74 \mathrm{~mm}$. Ce qui permet d'avoir une disponibilité en eau au sol nécessaire pour le développement et la croissance de l'espèce Anacardium occidentale dans la commune de Savalou (Yabi et al., 2013). Le relief est constitué d'une pénéplaine de $300 \mathrm{~m}$ d'altitude en moyenne. Cette pénéplaine est parsemée de collines dont l'altitude atteint $520 \mathrm{~m}$ avec des pentes variant entre 3 et $10 \%$ dans les sites agglomérés. Le milieu naturel de la commune de Savalou est composé des îlots de forêts denses, de savanes, de jachères et de champs. 
Le domaine des forêts denses a été transformé en plantations (agro-forêts), en vieilles jachères et en champs cultivés. Les sols les plus répandus sont les sols ferrugineux tropicaux avec par endroit des étendues de concrétion. On distingue aussi des sols hydromorphes et des vertisols.

La Commune de Savalou a une population de 104749 habitants, soit 50163 hommes et 54586 femmes, répartie dans quatorze (14) arrondissements dont quatre (04) urbains (Aga, Agbado, Attakè et Ouèssè) et dix (10) ruraux (Djalloukou, Doumè, Gobada, Kpataba, Lahotan, Lèma, Logozohè, Monkpa, Ottola et Tchetti). Les principaux groupes ethniques qui se partagent le territoire de la commune sont: le groupe Adja-Tado constitué des Fon et des Mahi (58\%), le groupe Yoruba et apparentés constitué des Ifè, des Itcha et des Idaacha (32\%) et le groupe ethnique issus des migrations récentes: ce sont les Yom-Lokpa (2,3\%), les Peulh (2,2\%), les Otamari $(2,5 \%)$, les Dendi et les Baatonu $(0,9)$ et autres $(2,5 \%)$.

Les activités économiques sont constituées des services commerciaux, les entreprises modernes, l'artisanat, le tourisme, l'hôtellerie et l'agriculture qui représente la principale activité. Les principales cultures réalisées dans la Commune sont l’igname, le manioc, le maïs, le riz, le niébé, l'anacarde et des cultures maraîchères.

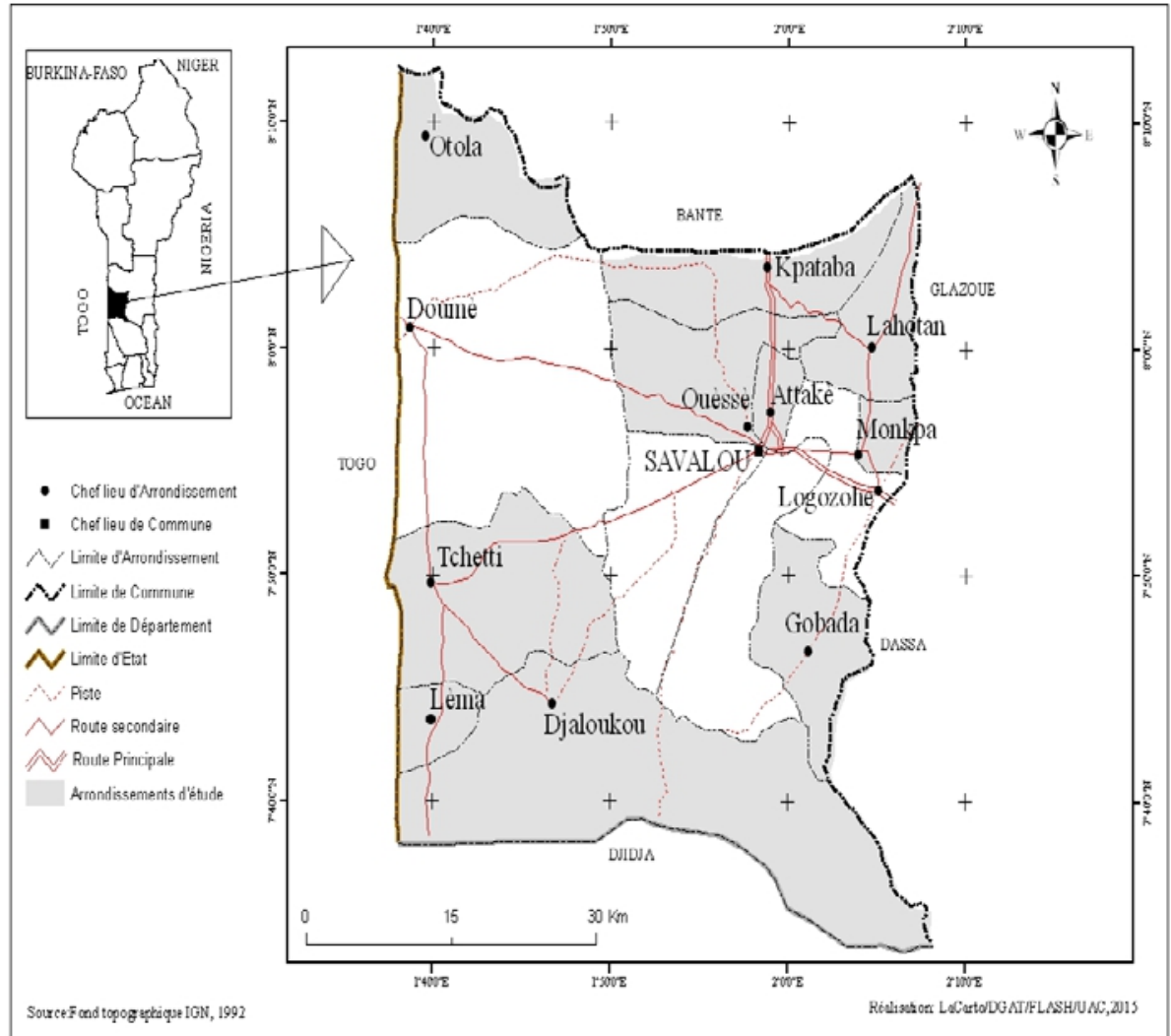

Figure 1: Localisation des arrondissements enquêtés dans la commune de Savalou au Bénin. 


\section{Choix Des Arrondissements D'etude}

Les producteurs enquêtés sont répartis par Arrondissement (tableau 1)

Tableau 1 : Répartition des producteurs enquêtés par arrondissement

\begin{tabular}{ccc}
\hline & Réparation par Arrondissement & \\
\hline Arrondissements & Effectifs & Pourcentage(\%) \\
\hline Ouèssè & 34 & 16,2 \\
Lahotan & 28 & 13,3 \\
Monkpa & 22 & 10,5 \\
Djalokou & 22 & 10,5 \\
Tchètti & 21 & 10,0 \\
Gobada & 12 & 5,7 \\
Lèma & 23 & 11,0 \\
Attakè & 13 & 6,2 \\
Ottola & 23 & 11,0 \\
Kpataba & 12 & 5,7 \\
\hline
\end{tabular}

\section{Methode echantillonnage}

La taille de l'échantillon pour la réalisation de l'enquête est déterminée par la formule de Dagnelie (1998). $n=U_{1-\alpha / 2}^{2} X \quad p(1-p) /$ $\mathrm{d}^{2}$

$\mathrm{n}=$ taille de l'échantillon ; ni = taille de l'échantillon par arrondissement ; $\mathrm{U}^{2}{ }_{1-\alpha / 2}$ est la valeur de la variable aléatoire normale pour une valeur de probabilité de $1-\alpha / 2=0,975 ; \alpha=0,05 ; \quad \mathrm{U}_{1-\alpha / 2}^{2}=1,96$; p est la proportion des planteurs d'anacardier ayant perçu l'effet du changement climatique sur la production de l'anacardier et d est la marge d'erreur d'estimation fixée à 0,05 dans cette étude. A partir de $\mathrm{P}=0,7$ issues des résultats de la phase exploratoire de l'étude, au total 210 producteurs ont été sélectionnés dans le milieu d'étude et répartis dans 10 Arrondissements (Tableau1).

\section{Methodes et outils de collecte des donnees}

Un questionnaire d'enquête est adressé aux propriétaires de plantation d'anacardier de décembre 2014 à février 2015. Ce qui a permis de recueillir des informations quantitatives et qualitatives. Les types de données collectées sont relatifs aux caractéristiques socioculturelles des planteurs d'anacardier, aux contraintes de la production, aux effets du changement climatique et aux causes de baisse de rendement des plantations d'anacardiers. Les méthodes d'adaptation utilisées par les planteurs d'anacardier pour atténuer les effets des changements climatiques ont été identifiées. Quant aux données pluviométriques, elles ont été collectées dans le SCDA de Savalou et à l'ASECNA sur 32 ans (soit de 1982 à 2014) 


\section{Traitement Et Analyse Des Donnees}

Pour l'analyse des données issues de l'enquête, après codification dans le tableur Microsoft Excel, les données ont été analysées à l'aide du logiciel SPSS (Statistical Package for the Social Sciences) version 16.0 pour la détermination des statistiques descriptives en termes de pourcentage et de moyenne. Les données quantitatives ont été ensuite soumises à une analyse de variance (ANOVA) (Balogoun et al., 2014) en utilisant la procédure PROC GLM du logiciel SAS (Statistical Analysis System) version 9.2 afin de déterminer les variations d'un arrondissement à un autre et d'un groupe socioculturel à l'autre. Les comparaisons de moyennes multiples ont été réalisées avec le test de la plus petite différence significative (ppds) à 5\%.

Les sujets enquêtés ont été regroupés suivant les quatre principaux groupes socioculturels, à savoir les Idaatcha, Mahi, Nago et Ifè. Dans chaque groupe, les planteurs enquêtés ont été regroupés suivant trois catégories d’âge en tenant compte de la classification effectuée par Assogbadjo et al. (2008) et Gnanglè et al. (2011), à savoir : Jeune = inférieur à 50 ans ; adulte $=50$ à 70 ans ; vieux = Plus de 70 ans). Ainsi, au total, 10 catégories socioculturelles ont été considérées. La situation relative aux 10 catégories socioculturelles au lieu des 24 potentielles résulte de l'absence dans l'échantillon étudié de certains enquêtés répondant à des combinaisons de modalité d’âge, de groupes ethniques et surtout de l'absence des femmes propriétaires de plantation d'anacarde.

Pour analyser les perceptions des producteurs par rapport aux problèmes rencontrés ces dernières années, l'effet des facteurs climatiques sur la productivité de l'anacardier et les causes de baisse de rendement, les différents groupes sociolinguistiques ont été mis en relation avec les paramètres de perception grâce à une analyse en composantes principales (ACP) selon Uguru et al. (2011), Bello (2014) pour faciliter l’interprétation des résultats.

Par une démarche analytique, les méthodes d'adaptation aux changements climatiques utilisées par les différents groupes socioculturels ont été identifiées. Pour chaque groupe, le nombre de planteur d'anacardier ayant opté pour chacune des méthodes a été calculé. Le tableau de contingence obtenu après avoir filtré et trié les groupes, a été soumis à une analyse factorielle des correspondances simple (AFC). Les analyses ACP et AFC ont été réalisées grâce au logiciel MINITAB14 (Minitab, 1996).

Pour mettre en évidence le caractère évolutif de la pluviométrie de la Commune de Savalou entre 1982 et 2014 les données pluviométriques ont été analysées avec le logiciel Minitab 14 en tenant compte de certains paramètres de précision comme l'erreur moyenne absolue en pourcentage (MAPE), la déviation moyenne absolue (MAD) et la déviation moyenne quadratique (MSD). 


\section{Résultats}

\section{Caractéristiques des planteurs et plantations d'anacardier dans la Commune de Savalou}

D’une manière générale et selon les résultats de l'enquête, il y a une variation entre les différents paramètres mesurés. Le tableau 2 présente les résultats de l'analyse descriptive des variables socioculturelles des propriétaires de vergers dans la commune de Savalou. Les hommes constituent la couche détentrice des plantations d'anacardier $(100 \%$ des personnes enquêtées sont de sexe masculin). Dans la commune d'étude l'âge de la majorité des planteurs est inférieur à 50 ans (80\%). La plus part des planteurs de la commune sont non instruits. La majorité des plantations sont détenues par les Mahi (71,4\%). Le groupe sociolinguistique Idaatcha a moins de plantation dans la Commune de Savalou. La densité de l'ensemble des plantations de la Commune est supérieure à la norme (100 plants/ha) et varie d'un arrondissement à un autre.

Tableau 2: Caractéristiques des planteurs et plantations de la Commune de Savalou

\begin{tabular}{cccc}
\hline Variables & Modalité & Fréquence absolue & Fréquence relative (\%) \\
\hline \multirow{2}{*}{ Age } & $<50$ ans & 168 & 80 \\
& 50 à 70ans & 39 & 18,6 \\
& $>70$ ans & 3 & 1,4 \\
\hline \multirow{2}{*}{ Niveau } & Non Instruit & 175 & 83,3 \\
d'éducation & Primaire & 13 & 6,2 \\
& Secondaire & 22 & 10,5 \\
\hline \multirow{2}{*}{ Ethnie } & Mahi & 150 & 71,4 \\
& Idaatcha & 11 & 5,2 \\
& Nago & 18 & 8,6 \\
& Ifè & 31 & 14,8 \\
\hline \multirow{2}{*}{ Densité des } & $<100$ & 45 & 21,4 \\
plantations & 100 à 125 & 77 & 36,7 \\
& $>125$ & 88 & 41,9 \\
\hline \multirow{2}{*}{ Age des } & $5<10$ ans & 38 & 18,1 \\
plantations & $10<$ 25ans & 146 & 69,5 \\
& $>25 \mathrm{ans}$ & 26 & 12 \\
\hline \multirow{2}{*}{ Superficie des } & $1<5$ ha & 77 & 36,7 \\
plantations & $5<10$ ha & 71 & 33,8 \\
& $>10$ ha & 62 & 29,5 \\
\hline
\end{tabular}

L'âge des planteurs et les superficies des plantations d'anacardier de la Commune ne sont pas significativement $(\mathrm{P}>0,05)$ différents d'un arrondissement à l'autre (Tableau 3). Néanmoins l'âge des plantations varie d'un arrondissement à un autre.

Les plantations les plus âgées se retrouvent successivement à Ottola, Ouèssè, Lèma et Lahotan. Le rendement moyen le plus élevé $(544,57 \mathrm{~kg} / \mathrm{ha})$ et le rendement moyen le plus bas $(386,15 \mathrm{~kg} / \mathrm{ha})$ sont respectivement obtenus dans les arrondissements d'Ottola et d'Attakè. Tableau 3: Données 
quantitatives (valeurs moyennes \pm erreur type) sur les plantations d'anacardier dans les Arrondissements d'étude.

\begin{tabular}{ccccc}
\hline Arrondissement & $\begin{array}{c}\text { Age des } \\
\text { planteurs } \\
\text { (an) }\end{array}$ & $\begin{array}{c}\text { Superficie des } \\
\text { plantations } \\
\text { (ha) }\end{array}$ & $\begin{array}{c}\text { Age des plantations } \\
\text { (an) }\end{array}$ & $\begin{array}{c}\text { Rendement } \\
\text { moyen avant } \\
2014(\mathrm{~kg} / \mathrm{ha})\end{array}$ \\
\hline Djalouko & $43,64 \pm 2,69 \mathrm{a}$ & $8,93 \pm 1,25 \mathrm{a}$ & $17,50 \pm 1,00 \mathrm{ab}$ & $336,36 \pm 9,97 \mathrm{ba}$ \\
Ottola & $43,78 \pm 2,38 \mathrm{a}$ & $9,87 \pm 1,46 \mathrm{a}$ & $20,65 \pm 1,76 \mathrm{a}$ & $325,22 \pm 13,76 \mathrm{~b}$ \\
Tcheti & $39,62 \pm 1,52 \mathrm{a}$ & $7,29 \pm 1,14 \mathrm{a}$ & $17,29 \pm 1,40 \mathrm{ba}$ & $346,43 \pm 12,11 \mathrm{ba}$ \\
Attakè & $44,46 \pm 2,17 \mathrm{a}$ & $9,77 \pm 2,06 \mathrm{a}$ & $16,92 \pm 1,62 \mathrm{ba}$ & $332,75 \pm 22,96 \mathrm{ba}$ \\
Gobada & $42,92 \pm 3,50 \mathrm{a}$ & $10,00 \pm 3,02 \mathrm{a}$ & $14,83 \pm 1,61 \mathrm{~b}$ & $332,75 \pm 22,96 \mathrm{ba}$ \\
kpataba & $39,17 \pm 2,42 \mathrm{a}$ & $8,00 \pm 1,38 \mathrm{a}$ & $15,58 \pm 1,48 \mathrm{~b}$ & $372,92 \pm 25,27 \mathrm{a}$ \\
Lahotan & $40,07 \pm 1,63 \mathrm{a}$ & $7,64 \pm 1,10 \mathrm{a}$ & $18,36 \pm 1,55 \mathrm{ba}$ & $362,53 \pm 15,60 \mathrm{ba}$ \\
Lèma & $43,39 \pm 1,58 \mathrm{a}$ & $8,08 \pm 1,06 \mathrm{a}$ & $18,70 \pm 1,77 \mathrm{ba}$ & $356,87 \pm 18,28 \mathrm{ba}$ \\
Monkpa & $42,41 \pm 1,66 \mathrm{a}$ & $7,77 \pm 1,38 \mathrm{a}$ & $15,23 \pm 1,21 \mathrm{~b}$ & $331,82 \pm 15,62 \mathrm{ba}$ \\
Ouèssè & $43,38 \pm 1,91 \mathrm{a}$ & $10,59 \pm 1,53 \mathrm{a}$ & $19,12 \pm 1,31 \mathrm{ba}$ & $330,29 \pm 13,74 \mathrm{ba}$ \\
\hline
\end{tabular}

Les moyennes suivie de la même lettre et dans la même colonne ne sont pas significativement différentes

( $\mathrm{P}>0,05)$ selon le test de la plus petite différence significative (ppds).

\section{Niveau de rendement}

La figure 2 présente les rendements moyens et la densité des arbres en 2014. La densité et le rendement obtenu en 2014 sont significativement différents $(p<0,05)$ entre l'arrondissement selon le test de la plus petite différence significative. Il ressort de l'analyse de la figure qu'en 2014, le rendement moyen le plus élevé $(372,92 \mathrm{~kg} / \mathrm{ha})$ est obtenu dans l'arrondissement de kpataba ; le rendement moyen le plus faible et la densité la plus faible sont obtenu dans l'arrondissement d'Ottola.

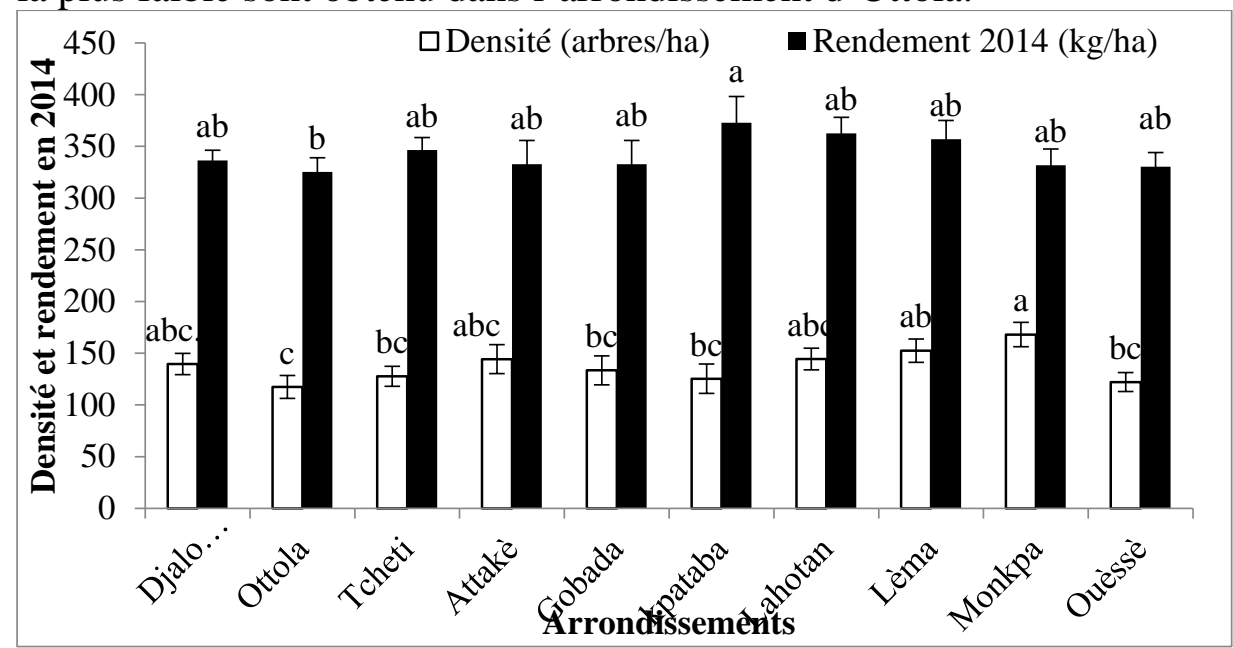

Figure 2 : rendement et densité en 2014

Perception des planteurs sur des contraintes de production de l'anacardier

Les proportions de réponses liées aux problèmes auxquels sont confrontés les planteurs d'anacardier de la commune de Savalou ces 
dernières années sont présentées par la Figure 3. L’analyse indique que c'est la sécheresse prolongée qui constitue la principale cause de baisse de productivité suivi de l'harmattan.

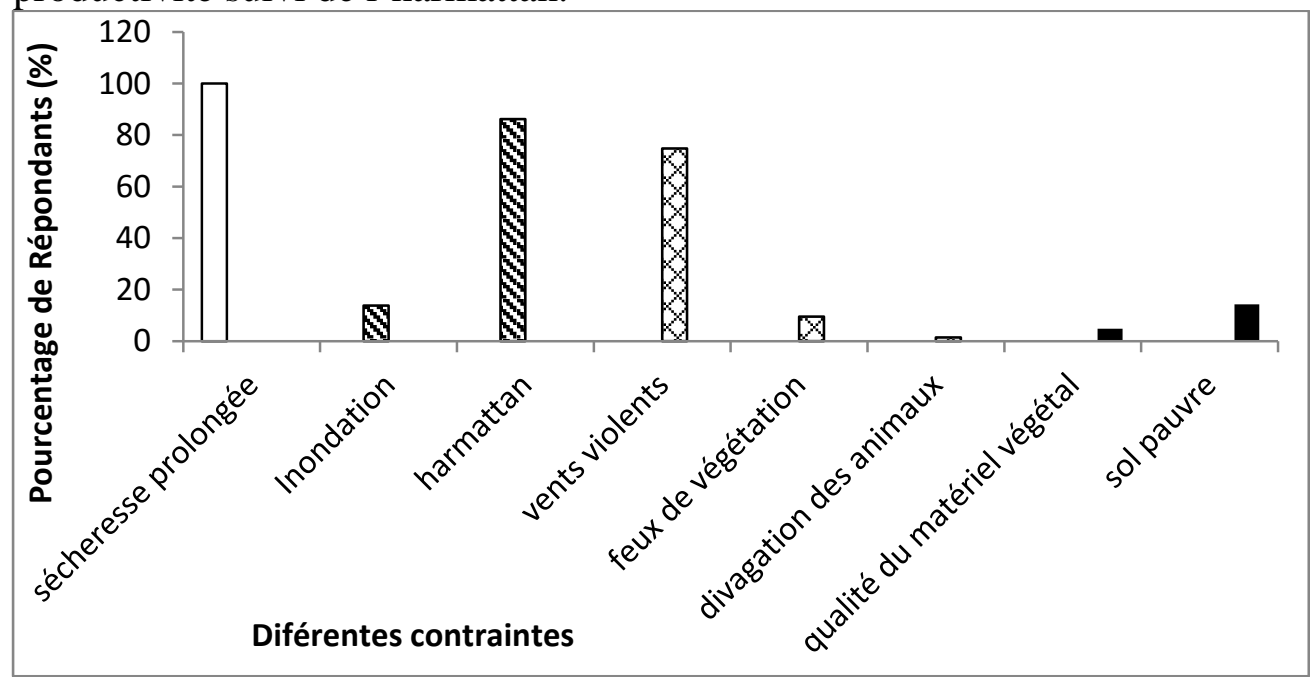

Figure 3 : Perception des différentes contraintes de production.

La projection des 10 catégories socioculturelles dans le système d'axe formé par les différentes contraintes de production (Figure 4) révèle que les adultes et les jeunes des groupes socioculturels Nago, Mahi, et Ifè ont la perception que ce sont les changements climatiques qui ont été les contraintes de production d'anacarde tandis que les vieux Ifè et Mahi pensent que ce sont plutôt la divagation des animaux et les feux de végétation ainsi que la densité des arbres. Quant aux jeunes et adulte Idatcha, ils perçoivent plutôt que ce sont la mauvaise qualité du matériel végétal et le mauvais entretien des plantations qui constituent les problèmes majeurs de production d'anacarde. 

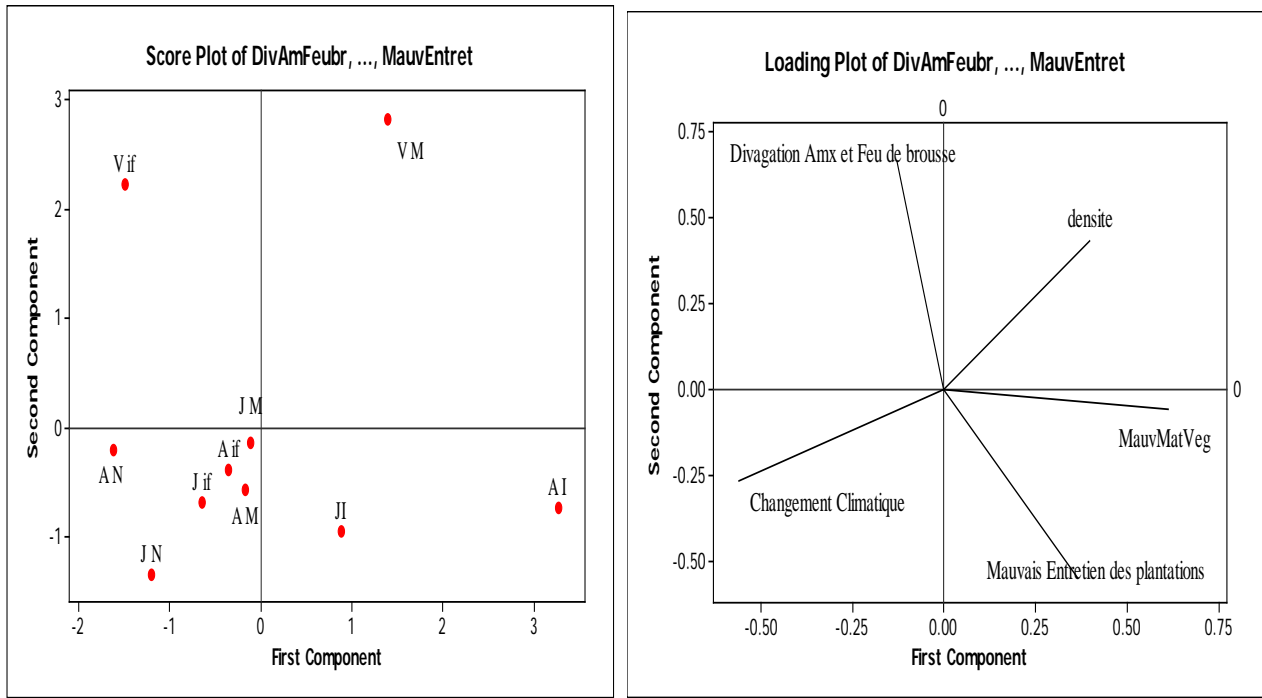

Figure 4: Perceptions des producteurs des contraintes de production de l'anacardier étudiées à partir d'une Analyse en Composantes Principales (ACP) : projection des contraintes et des groupes socio-culturels dans le système d'axes factoriels.

Légende : Adulte Idaatcha=AI; Adulte Ifè = Aif ; Adulte Mahi=AM; Adulte Nago

$=A N ;$ Jeune Idaatcha $=J I$; Jeune Ifè=Jif ; Jeune Mahi=JM ; Jeune Nago=JN ; Vieux Ifè=Vif ; Vieux Mahi $=$ VM ; MauvMatVeg : Mauvais matériel végétal

Perception des producteurs sur les évènements climatiques affectant la productivité des anacardiers dans la Commune de Savalou

La figure 5 présente les proportions de réponses liées aux évènements climatiques affectant la productivité d'anacardier dans la commune de Savalou. L'analyse de la figure 4 indique que c'est le déficit de pluie qui est le principal paramètre climatique affectant la production d'anacarde suivie de la mauvaise répartition des pluies et l'harmattan de longue durée. L'harmattan réduit la floraison et entraine la coulure des fleurs après les avoir séchés. Selon les planteurs d'anacardier les excès de pluies ne font qu'augmenter le rendement car la période de production ne coïncide pas avec la saison pluvieuse. L'inondation et l'humidité ne coïncident pas avec la période de fructification pour avoir d'impact négatif (autres que la prolifération des ravageurs) sur la production des noix. Ainsi la réserve utile d'eau contenu dans le sol au cours des années d'excès de pluies permettent à la plante de mieux produire. 


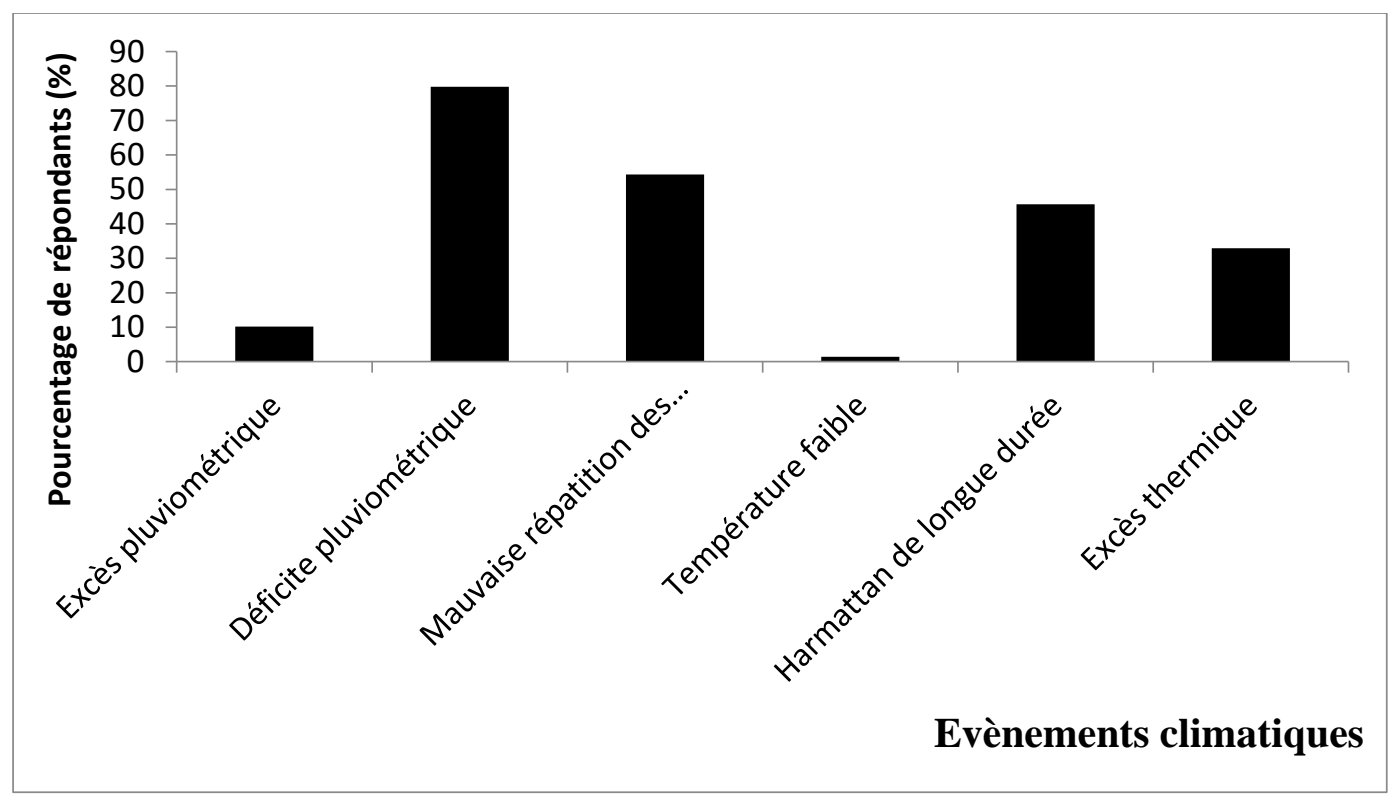

Figure 4: Perception des différents évènements climatiques.

Les résultats de l'analyse en composante principale sur les différents groupes socioculturels et les différents évènements climatiques ont permis de décrire les relations entre ces perceptions des évènements climatiques et d'affiner leur analyse. Ces résultats indiquent que les deux premiers axes expliquent 81,5 \% de l'information totale (Figure 6). La première composante oppose l'excès de pluie, la mauvaise répartition des pluies et l'excès thermique aux périodes d'apparition de l'harmattan, à l'harmattan de courte durée et à l'harmattan de longue durée. La seconde composante par contre oppose le déficit pluviométrique à la basse température. Selon les populations, un déficit pluviométrique est souvent accompagné d'une chaleur excessive.

La projection des 10 catégories socioculturelles dans le système d'axe formé par les évènements climatiques révèle que les adultes et les jeunes des groupes socioculturels Nago et Ifè ont la perception que ce sont les périodes d'apparition de l'harmattan et sa durée qui sont les évènements climatiques qui affectent la production d'anacarde tandis que les vieux Ifè et Mahi et les jeunes, Idaatcha pensent que ce sont plutôt le déficit pluviométrique, la mauvaise répartition des pluies et l'excès thermique qui sont les évènements climatiques qui affectent la production des noix d'anacardier dans la Commune de Savalou. 

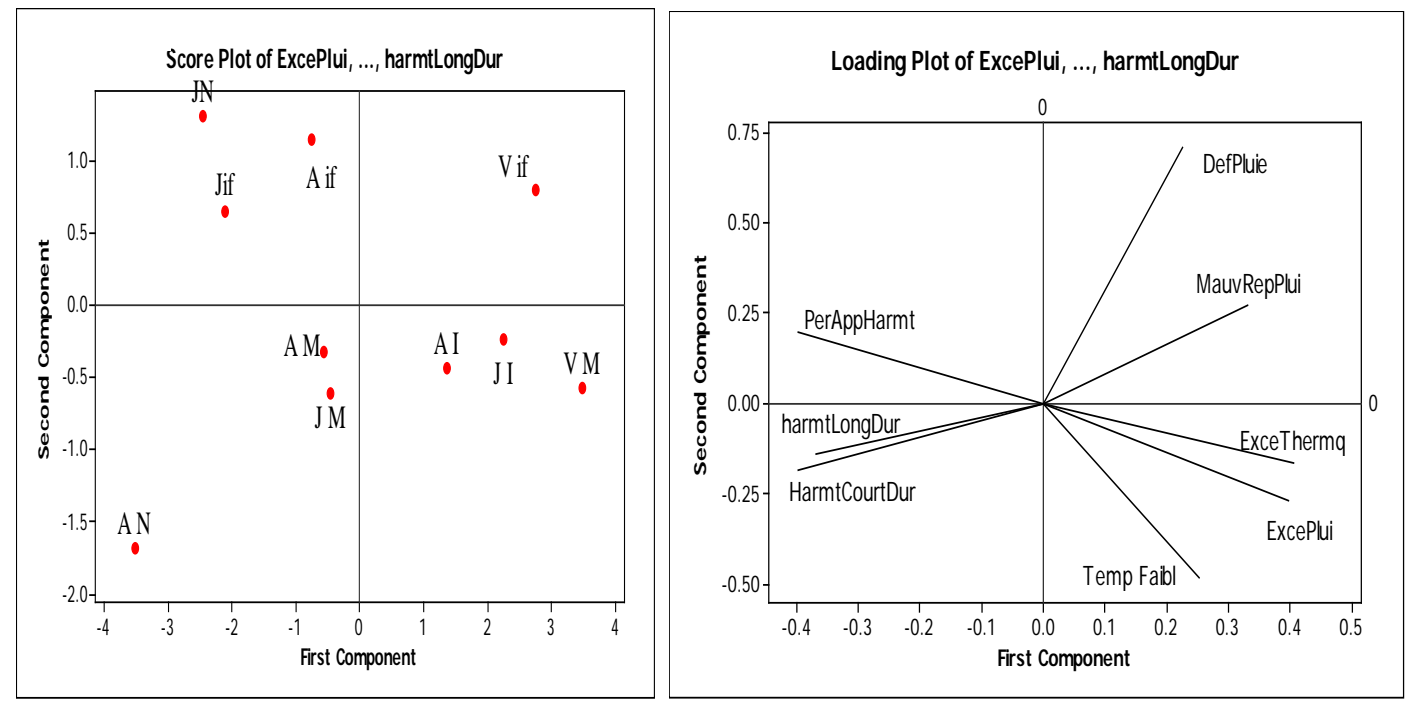

Figure 6: Perceptions des producteurs sur les évènements climatiques affectant la production de l'anacardier étudiés à partir d'une Analyse en Composantes Principales (ACP) : projection des évènements climatiques et des groupes socio-culturels dans le système d'axes factoriels.

Légende : Adulte Idaatcha $=A I$; Adulte Ifè $=$ Aif ; Adulte Mahi $=A M$; Adulte Nago $=A N$; Jeune Idaatcha=JI ; Jeune Ifè=Jif ; Jeune Mahi=JM ; Jeune Nago=JN ;

Vieux Ifè=Vif ; Vieux Mahi=VM ; Déficit pluviométrique = DefPluie ; Mauvaise répartition des pluies $=$ MauvRepPlui $;$ Excès thermique $=$ ExceThermq $;$ basse température = TempFaibl ; Période d'apparition de l'harmattan= PerAppHarmt ; Harmattan de courte durée = HarmtCourtDur ; Harmattan de Longue Durée = harmtLongDur

\section{Stratégies d'adaptation aux effets du changement climatique}

La figure 7 présente les fréquences relatives liées aux stratégies d'adaptations. L'analyse de la figure montre que selon les propriétaires de verger, le labour suivi du fauchage à la fin des saisons pluvieuses est la principale stratégie d'adaptation aux changements climatiques utilisée dans les plantations d'anacardier. Cette stratégie permet de réduire l'évapotranspiration du sol car le labour crée des espaces encaissés pour retenir de l'eau et les adventices fauchés constituent un mulch. Ensuite viennent l'éclaircie et l'élagage suivi d'entretien qui contribuent à une réduction de la concurrence en éléments nutritifs et de photosynthèse. 
Figure 7: Stratégies d'adaptations

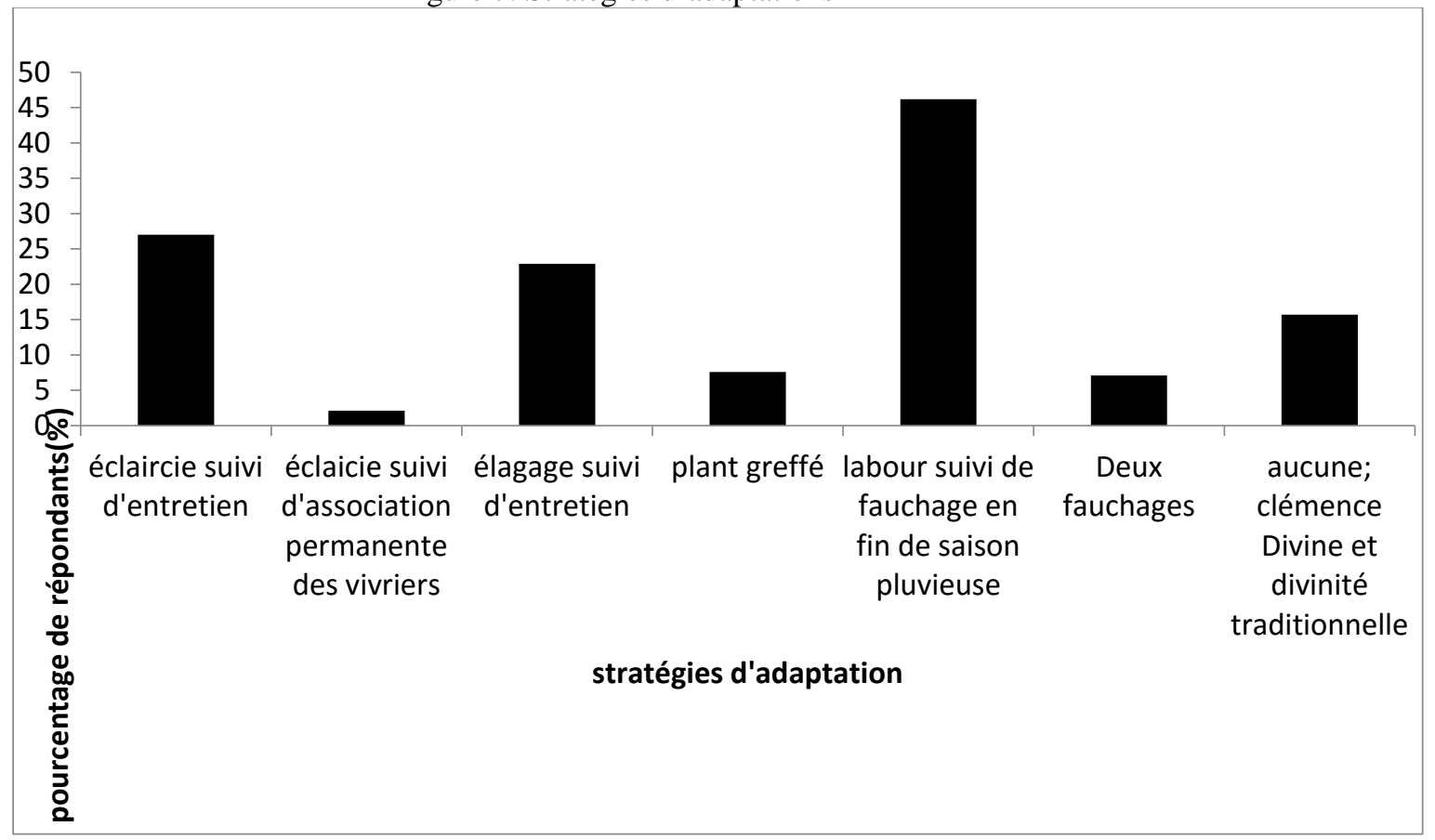

La Figure 8 présente les résultats de l'analyse factorielle des correspondances simple réalisés sur les méthodes d'adaptations et montre que les vieux Mahi et les jeunes Ifè adoptent l'éclaircie suivi d'association culturales permanentes, du labour suivi de fauchage et deux fauchages par an tout en implorant la clémence Divine. Les vieux Ifè, les jeunes idatcha et les adultes des groupes socioculturels Mahi et Nago ont compris que les plants greffés pourront résistés aux changements climatiques. Les adultes Idatcha pratiquent l'éclaircie au besoin qui réduit la densité de la plantation tandis que les jeunes Nago adoptent l'élagage pour atténuer les effets des changements climatiques. 


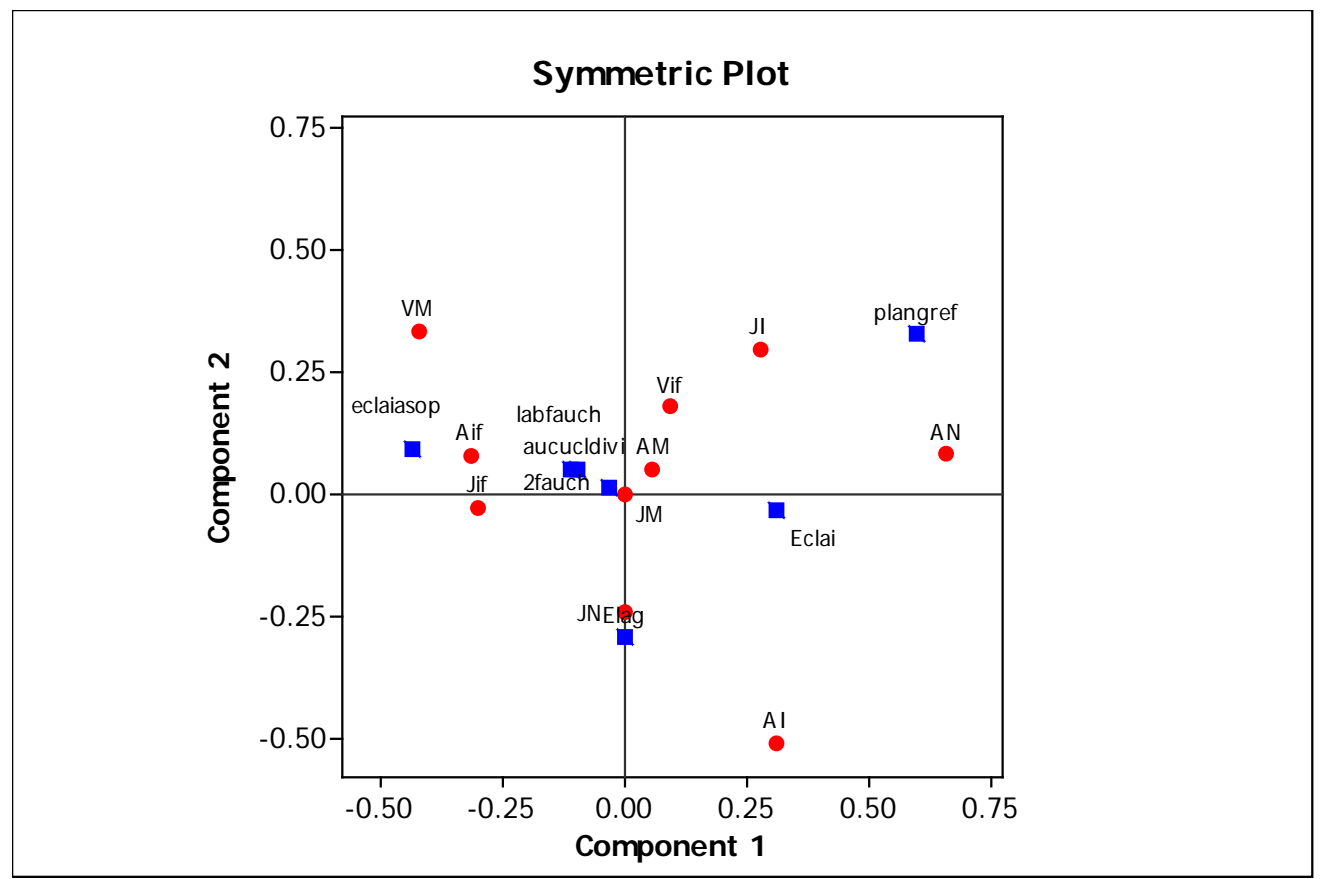

Figure 8: Adaptation face aux changements climatiques projection des groupes socioculturels dans le système d'axes factoriels à l'issue d'une Analyse Factorielle des Correspondances ; les codes des groupes socioculturels sont les mêmes que ceux utilisés en figure 3.

Légende : eclaiasop = éclaircie suivi d'association permanentes des vivriers ; labfauch= labour suivi de fauchage en fin de saison pluvieuse ; 2 fauch= deux fauchages ; eclai=éclaircie suivi d'entretien; Elag= élagage ; aucucldivi= aucune pratiques clémence divine.

\section{Caractéristiques des plantations d'anacardier}

Les résultats des travaux de recherche montrent que les hommes constituent la couche détentrice des plantations d'anacardier $(100 \%$ des personnes enquêtées). Pour ce qui est de l'absence des femmes, les règles coutumières restrictives des droits fonciers sur les terres au genre féminin pourraient constituer la cause principale de l'absence des planteurs femmes dans l'ensemble des arrondissements de Savalou. En effet, seuls les hommes peuvent hériter des terres des parents et les transmettre à leur tour à d'autres progénitures de sexe masculin. C'est ce que confirment les résultats des travaux de Tandjiékpon (2005), réalisés en zone de savane qui expliquent que les femmes accèdent le plus souvent à une portion de terres de leur mari pour la pratique des cultures saisonnières. Cette portion de terre, souvent dégradée et pauvre, concédée par l'époux est généralement de taille très modeste pour être valorisée avec des plantes pérennes. D’autres auteurs notamment ICA (2010) et Balogoun et al. (2014) ont également obtenu les mêmes résultats au centre et au nord-Ouest du Bénin. En Tanzanie un 
inventaire des propriétaires de plantations d'anacardiers a montré que les femmes représentent en moyenne $13 \%$ dans le sud du pays et $14 \%$ dans le nord (Topper et al., 2003). Selon l'auteur, bien que ce taux soit strictement supérieur à celui de la commune de Savalou, il reste néanmoins bas comparativement à la proportion des hommes qui sont impliqués. Les résultats de nos travaux de recherche montrent que les personnes ayant un âge inférieur à 50 ans détiennent plus de plantation que les autres classes d’âge. Ce résultat corrobore celui obtenu par Topper et Kasuga (2003), en Tanzanie où l'âge moyen des chefs d'exploitation des plantations d'anacardier est compris entre 44 et 51 ans dans la région sud du pays. Au Mozambique, une typologie des petits planteurs d'anacardier a montré que l'âge moyen du chef d'exploitation est de 48 ans. Ces âges moyens observés au Mozambique et en Tanzanie sont similaires à celui enregistré au Bénin (48 ans, Tandjiekpon, 2005 ; et 49 ans, Balogoun et al., 2014), dans les principales zones de production d'anacardier . Nos résultats montrent des âges inférieurs à ceux obtenus par Lawal et al. (2010) au niveau des planteurs d'anacardiers nigérians qui sont âgés de 56 ans en moyenne. Les densités de plant pratiquées varient de 50 à 250 plants par hectare (en moyenne 150 plants à l'hectare) (Tandjiekpon, 2005) et semblent être similaire à celle de la densité des plantations de la commune de Savalou (27 à 285 plants à l'hectare ; en moyenne 156 plants à l’hectare).

Les rendements en noix de cajou varient selon les conditions écologiques et les techniques culturales. Dans la commune de Savalou, le rendement moyen obtenu en 2014 est de 342,79 kg à l'hectare. Ce résultat confirme celui obtenu en 2013 par Balogoun et al. (2014). Les rendements sont relativement très faibles dans les trois zones de production d'anacarde (moins de $300 \mathrm{~kg} / \mathrm{ha}$ ). Or, selon Adégbola et al. (2005), les rendements moyens en noix de cajou au Bénin oscillent entre 350 et $600 \mathrm{~kg} / \mathrm{ha}$ avec quelques rares pics entre 1000 et $1500 \mathrm{~kg} / \mathrm{ha}$. Le rendement en noix d'anacardier obtenu dans la commune de Savalou est légèrement inférieur à celui obtenu par Sèdjro et Sanni-Agata (2002), qui ont montré qu’au Bénin, les rendements estimés sont généralement dans la fourchette de 350 à 600 $\mathrm{kg} / \mathrm{ha}$.

\section{Perception des planteurs sur l'effet des évènements climatiques}

La majorité des planteurs perçoivent les changements climatiques comme la contrainte majeure de la production d'anacarde. Ce résultat est en accord à celui obtenu par Bello (2014), qui a montré qu'aux dires des producteurs, les facteurs climatiques ont eu des effets négatifs sur les paramètres phénologiques et de rendement des plants d'anacardier en 2013. Dans la commune de Savalou, les adultes et les jeunes des groupes socioculturels Nago et Ifè perçoivent la période d'apparition de l’harmattan 
et sa durée comme des évènements climatiques qui affectent la production d'anacardier tandis que les vieux Ifè, Mahi et les jeunes Idaatcha pensent que ce sont plutôt le déficit pluviométrique, la mauvaise répartition des pluies et l'excès thermique qui constituent les causes de baisse de rendement. Les perceptions des planteurs diffèrent alors d'un groupe socioculturel et d'une classe âge à l'autre. Ces résultats sont en accord avec ceux de Yabi et Afouda (2012), qui ont montré que, la variabilité des facteurs climatiques est perçue et comprise de façon diverse par les populations africaines selon leurs caractéristiques socioculturelles. La majorité des vieux des quatre groupes socioculturels ont perçu que ce sont les changements climatiques qui affectent le rendement. Selon Oyekale et Oladele (2012), avec l’âge, l'habilité à percevoir les changements climatiques augmentait et chez les communautés rurales africaines les hommes perçoivent mieux les changements climatiques, probablement du fait de leur expérience et de leur contact avec le milieu extérieur.

L’instabilité des rendements s’explique par la variabilité climatique car l'arrondissement d'Ottola qui a le rendement moyen le plus élevé obtient le plus bas en 2014. Les évènements climatiques ont contribué à la baisse du rendement des anacardiers au niveau de tous les arrondissements de la Commune en 2014 (rendement passé de 544,57 à 325,22kg/ha). Selon les producteurs, les dernières pluies de la saison pluvieuse impactent le rendement en noix d'anacarde. Ceci confirme les analyses de Yabi (2008), selon lesquelles la pluviométrie des mois de septembre et d'octobre constitue une ressource hydrique précieuse pour l'anacardier qui l'utilise pendant les phases phénologiques les plus importantes (floraison et fructification) au cours des mois suivants.

Perception des planteurs sur les méthodes d’adaptation aux changements climatiques.

Certains planteurs d'anacardier de Savalou ont perçu que l'éclaircie suivi d'association permanentes des vivriers, le labour suivi de fauchage, deux fauchages par an, l'éclaircie suivi d'entretien et l'élagage contribuent à atténuer les effets des évènements climatiques. L’éclaircie et l'élagage contribuent à la réduction des concurrences en éléments nutritifs et photosynthétique. L'ensemble de ces méthodes d'adaptations confirme l'un des résultats de Tandjiékpon (2005), définissant l'importance du labour qui crée des espaces encaissés pour retenir de l'eau et de l'humus exploitables par les plants d'anacardier. En ce qui concerne les plants greffés, et pour les producteurs ayant expérimenté les deux méthodes de plantation, 58,91\% estiment que les plantations d'anacardier réalisées à base de plants greffés sortis de pépinière connaissent un meilleur développement végétatif comparativement aux plants issus du semis direct pour plusieurs raisons (Tandjiékpon, 2005). Ce qui confirme le choix de certains planteurs 
d'anacardier de Savalou. D’autres raisons sont avancées au nombre desquelles on peut citer le bon enracinement des plants du fait de la trouaison et la bonne reprise des plants après la plantation. Or un bon enracinement des plantes entrainerait une grande exploration des terres, une bonne nutrition et un bon rendement. Selon Lawson (2012), pour l'adaptation aux effets du changement climatique, il est nécessaire de mettre au point de nouveaux matériels végétaux ou de nouvelles variétés qui pourraient supporter les nouvelles conditions climatiques du milieu. De ce point de vue, l'amélioration du matériel végétal reste un axe majeur et intéressant à aborder par les questions de recherche.

Contrairement à toutes ces méthodes d'adaptations aux changements climatiques, d'autres planteurs d'anacardier de Savalou n'adoptent aucune pratique et n'implorent que la clémence divine. Ce qui rejoint les résultats des travaux de Yabi (2008), qui expliquent que les planteurs d'anacardier font preuve de passivité devant les contraintes climatiques en ce qui concerne la culture de l'anacardier. Ils n’ont pas de réponses adéquates face aux effets de la variabilité et des changements climatiques sur la production de l'anacardier. Il urge donc que les réflexions soient orientées vers les stratégies d'atténuation des effets du changement climatique.

\section{Conclusion}

La présente étude réalisée a permis d’identifier la perception des producteurs d'anacardier de la Commune de Savalou sur les effets des changements climatiques. Il ressort des résultats de cette étude que les changements climatiques affectent la production des plantations d'anacardier selon les producteurs dans la Communes de Savalou. La sécheresse prolongée suivi d’un déficit pluviométrique, la mauvaise répartition des pluies et l'harmattan sont des évènements climatiques les plus enregistrés et constituent les contraintes majeures à la production des anacardiers dans la Commune d'étude. Les autres causes qui entrainent la baisse de rendement des anacardiers dans la Commune de Savalou sont constituées de la divagation des animaux et du mauvais matériel végétal utilisé par les propriétaires de verger d'anacarde. Les travaux de cette recherche ont permis d’identifier les méthodes d'atténuation adoptées par les propriétaires des plantations d'anacardier de la Commune de Savalou.

\section{References:}

Adégbola P. Y., Oloukoï L. et H. C. Sossou. 2005. Analyse de la compétitivité de la filière anacarde au Bénin. Rapport technique final, PAPA/INRAB, Bénin, 35 p.

Aïvodji J. Anasside A., 2009. Elaboration des règles de stabilisation et de soutien des prix pour la filière anacarde, 78p. 
Assogbadjo A., E, Glèlè kakaï R., Chadare F, J., Thomson L., Kyndt T., Sinsin B., et Van Damme P. 2008. Folk classification, perception and preferences of baobab products in West Africa: consequences for species conservation and improvement. Economic Botany, 62(1): 74-84.

Balogoun I., Saïdou A., Ahoton E. L. Amadji G, L., Ahohuendo C. B., Adebo J. B., Babatoundé S., Chougourou D., Adoukonou S. H., Ahanchédé. 2014. Caractérisation des systèmes de production à base d'anacardier dans les principales zones de culture au Bénin. Agronomie Africaine 26 (1) : 1 14.

Bello O. D. 2014. Effet des facteurs climatiques sur la productivité de l'anacardier au Bénin. Mémoire pour l’obtention du Diplôme d'Etudes Approfondies (DEA). FSA/UAC, 86p.

Djenontin A. A. M. 2013. Perception des producteurs sur les facteurs climatiques affectant la productivité de l'anacardier dans la commune de Djougou au Benin, Mémoire Pour l’obtention du Diplôme de Licence Professionnelle à la FSA/UAC, Abomey-Calavi, Bénin, 82p.

FAO, 2014. Base des données de la FAO 2011. http://faostat3.fao.org.,

Gnanglè C. P. 2012. Perceptions paysannes du changement climatique : stratégies d'adaptation dans la gestion des parcs à karité au Bénin. Thèse de Doctorat unique en Sciences Agronomiques de l'Universite d'AbomeyCalavi, 154p.

Gnanglè P.C., Glèlè Kakaï R. L., Assogbadjo A. E., Vodounon S., Yabi J. A., Sokpon N. 2011. Tendances climatiques passées, modélisation, perceptions et adaptations locales au Bénin. Climatologie, 26-40.

Groupe de Discussion Africa Adapt (GDAA). 2011. Agriculture et Changements Climatiques. Témoignages sur les Impacts Observés et les Recommandations pour l'Action Politique, 12p.

Jayathilaka P.M.S., Soni P., Perret S., Jayasuriya H.P.W.et Salokhe V.M. 2012. Spatial assessment of climate change effects on crop suitability for major plantation crops in Sri Lanka. Regional environmental change: 55-68. Lawal J. O., Oduwole O. O, Shittu T. R. and A. A. Muyiwa. 2010. Profitability of value addition to cashew farming households in Nigeria. African Crop Science Journal 19 (1) : 49 - 54.

Lawson R. G. 2012. Caractérisation morphologique d'arbres élites d'anacardier (Anacardium occidentale, Linnaeus) et essai d'amélioration du matériel végétal par greffage au Bénin. Thèse pour l’obtention du Diplôme d’Ingénieur Agronome à l'Université de Parakou à la Faculté d’Agronomie, 72p.

Mamoudou A. H. 2012. Effet des changements climatiques sur la production agricole et mesures d'adaptation de la population : cas de la commune de Says. (Niger) 64p. 
Métangbo D., Kouassi D., Brama K. 2007. Vulnérabilité de l'agriculture pluviale au changement de régime pluviométrique et adaptation des communautés rurales du « V-Baoulé » en Côte d'Ivoire, 11p.

Oyekale A. S., Oladele O. I. 2012. Determinants of climate change adaption among cocoa farmers in southwest Nigeria. Food, Agriculture and Environment (JFAE), 10(34): 1562- 1567. PDC, 2005. Plan de Développement Communal de Savalou, 146p. RAC-F, 2014. Adaptation de l'agriculture aux changements climatiques: recueil d'expériences territoriales, 59p.

Sèdjro M.A., Sanni-Agata L. 2002. Analyse du secteur de l'anacarde : Situation actuelle et perspective de développement. Projet No.INT/W3/69.Centre du Commerce International CNUCED/OMC (CCI). Cotonou, $38 \mathrm{p}$.

Tandjiekpon A. 2005. Caractérisation du système agroforestier à base d'anacardier (anacardium occidentale linnaeus) en zone de savane au Bénin. Thèse pour l'obtention du Diplôme d'Etudes Approfondies (DEA), 104p.

Tinlot M. 2010. Evaluation ex ante du bilan carbone des dynamiques de développement agricole et forestier: Application sur la filière anacarde au Burkina Faso. Mémoire de Fin d'Etudes d'ingénieur de l'Institut Supérieur d'Agriculture de LILLE, 89P.

Topper C., Kasuga L. J. 2003. Knowledge transfer for sustainable tree crop development. A case history of the Tanzanian integrated cashew management programme. BioHybrids Agrisystems Ltd. RG6 5FY, UK ISBN 0-9545192-0-5.

Uguru M. I., Baiyeri K. P., Aba S. C. 2011. Indicators of climate change in the derived savannah niche of Nsukka, South eastern Nigeria. Journal of Tropical Agriculture, Food, Environment and Extension, 10(1): 17-26.

Yabi I., Biaou F.Y., Dadegnon S. 2013. Diversité des espèces végétales au sein des agro-forêts à base d'anacardier dans la commune de Savalou au Benin International Journal of biological and Chemical Sciences 7(2): 696706.

Yabi I. 2008. Etude de l'agroforesterie à base de l'anacardier et des contraintes climatiques à son développement dans le centre du Bénin, 240p.

Yabi I., Afouda F. 2012. Extreme rainfall years in Benin (West Africa), Quaternary International, 262(7): 39-43. 